\title{
SIRFLOX: Randomized Phase III Trial Comparing First-Line mFOLFOX6 (Plus or Minus Bevacizumab) Versus mFOLFOX6 (Plus or Minus Bevacizumab) Plus Selective Internal Radiation Therapy in Patients With Metastatic Colorectal Cancer
}

Guy A. van Hazel, Volker Heinemann, Navesh K. Sharma, Michael P.N. Findlay, Jens Ricke, Marc Peeters, David Perez, Bridget A. Robinson, Andrew H. Strickland, Tom Ferguson, Javier Rodríguez, Hendrik Kröning, Ido Wolf, Vinod Ganju, Euan Walpole, Eveline Boucher, Thomas Tichler, Einat Shacham-Shmueli, Alex Powell, Paul Eliadis, Richard Isaacs, David Price, Fred Moeslein, Julien Taieb, Geoff Bower, Val Gebski, Mark Van Buskirk, David N. Cade, Kenneth Thurston, and Peter Gibbs

Listen to the podcast by Dr Ko at www.jco.org/podcasts

Author affiliations appear at the end of this article.

Published online ahead of print at www.jco.org on February 22, 2016

Written on behalf of the SIRFLOX Study Group. The principal investigators are listed in the online-only Appendix.

Processed as a Rapid Communication manuscript.

Supported by Sirtex Technology.

The protocol has been published (Gibbs et al. BMC Cancer 2014;14:897) and the results presented in part at the 2015 ASCO Annual Meeting, May 19-June 2, 2015, Chicago, IL.

Authors' disclosures of potential conflicts of interest are found in the article online at www.jco.org. Author contributions are found at the end of this article.

Clinical trial information: NCT00724503.

Corresponding author: Guy A. van Hazel, MBBS, 22/146 Mounts Bay Rd, Perth WA 6000, Australia; e-mail: gvh@ perthoncology.com.au

(C) 2016 by American Society of Clinical Oncology

0732-183X/16/3415w-1723w/\$20.00 DOI: $10.1200 / J C O .2015 .66 .1181$

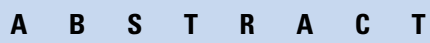

\section{Purpose}

SIRFLOX was a randomized, multicenter trial designed to assess the efficacy and safety of adding selective internal radiation therapy (SIRT) using yttrium-90 resin microspheres to standard fluorouracil, leucovorin, and oxaliplatin (FOLFOX)-based chemotherapy in patients with previously untreated metastatic colorectal cancer.

\section{Patients and Methods}

Chemotherapy-naïve patients with liver metastases plus or minus limited extrahepatic metastases were randomly assigned to receive either modified FOLFOX (mFOLFOX6; control) or mFOLFOX6 plus SIRT (SIRT) plus or minus bevacizumab. The primary end point was progression-free survival (PFS) at any site as assessed by independent centralized radiology review blinded to study arm.

\section{Results}

Between October 2006 and April 2013, 530 patients were randomly assigned to treatment (control, 263; SIRT, 267). Median PFS at any site was 10.2 v10.7 months in control versus SIRT (hazard ratio, 0.93; 95\% $\mathrm{Cl}, 0.77$ to $1.12 ; P=.43$ ). Median PFS in the liver by competing risk analysis was $12.6 v 20.5$ months in control versus SIRT (hazard ratio, 0.69; 95\% Cl, 0.55 to 0.90; $P=.002$ ). Objective response rates (ORRs) at any site were similar (68.1\% $v 76.4 \%$ in control $v$ SIRT; $P=.113)$. ORR in the liver was improved with the addition of SIRT (68.8\% $v 78.7 \%$ in control $v$ SIRT; $P=.042$ ). Grade $\geq 3$ adverse events, including recognized SIRT-related effects, were reported in $73.4 \%$ and $85.4 \%$ of patients in control versus SIRT.

\section{Conclusion}

The addition of SIRT to FOLFOX-based first-line chemotherapy in patients with liver-dominant or liveronly metastatic colorectal cancer did not improve PFS at any site but significantly delayed disease progression in the liver. The safety profile was as expected and was consistent with previous studies.

\section{J Clin Oncol 34:1723-1731. (C) 2016 by American Society of Clinical Oncology}

\section{INTRODUCTION}

Colorectal cancer (CRC) is the third most frequently diagnosed cancer in males and the second most frequent in females, with an estimated 1.4 million cases and 693,900 deaths occurring in 2012. ${ }^{1}$ The liver is the dominant site of metastatic disease in CRC, and an increasingly aggressive surgical approach to this patient population is leading to more long-term survivors. However, $80 \%$ to $90 \%$ of patients with liver metastases are not amenable to surgery at diagnosis, ${ }^{2-5}$ and liver metastases remain the dominant cause of death for patients with CRC. ${ }^{6-9}$

Many liver-directed therapies have been developed to control liver metastases or primary liver cancer, but no large phase III trials have been undertaken to fully assess the clinical usefulness 
of such therapies. Selective internal radiation therapy (SIRT), also known as radioembolization (SIR-Spheres ${ }^{\circledR}$, Sirtex Medical Limited, Sydney, Australia), ${ }^{10}$ delivers a single, measured, targeted radiation dose to liver tumors via injection into the hepatic artery. Yttrium-90 $\left({ }^{90} \mathrm{Y}\right)$-labeled resin microspheres have a median diameter of $32.5 \mu \mathrm{m}$, considerably smaller than the particles of other liver-directed therapies such as transarterial chemoembolization, which enables the microspheres to lodge distally within the microvascular plexus of tumors. ${ }^{11}$ Indeed, several authors have described SIRT as a form of liver-directed brachytherapy. ${ }^{10,12,13}$

Previous small studies have demonstrated that combining SIRT with first-line fluoropyrimidine-based chemotherapy increased objective response rates (ORRs) and extended time to progression and overall survival (OS) in patients with metastatic CRC (mCRC). ${ }^{14}$ A phase I study demonstrated that SIRT could be added safely to oxaliplatin-based chemotherapy, with promising outcome data. ${ }^{15}$ Given these data, the SIRFLOX study, a large randomized controlled trial of fluorouracil, leucovorin, and oxaliplatin (FOLFOX)-based chemotherapy with or without ${ }^{90}$ Y-labeled resin microspheres as first-line treatment of patients with liver-only or liver-dominant mCRC, was undertaken. ${ }^{16}$

\section{PATIENTS AND METHODS}

The SIRFLOX study was conducted in accordance with the Declaration of Helsinki, and approval was obtained from the relevant institutional review boards for each participating center. The study protocol has been described previously. ${ }^{16}$

\section{Patients}

Patients 18 years or older with histologically confirmed adenocarcinoma of the colon or rectum (with or without the primary tumor in situ) with proven liver metastases were enrolled. Patients had to be chemotherapy naïve for mCRC (previous adjuvant systemic chemotherapy for primary CRC or neoadjuvant chemoradiotherapy to the pelvis completed $>6$ months before recruitment was permitted), have a WHO performance status of 0 to 1 , and have a life expectancy of $\geq 3$ months. Patient inclusion and exclusion criteria are described in Appendix Table Al (online only).

\section{Study Design and Interventions}

SIRFLOX was a randomized, multicenter trial of systemic chemotherapy with modified FOLFOX (mFOLFOX6) plus or minus SIRT as firstline treatment of patients with nonresectable liver-only or liver-dominant mCRC. Liver-dominant mCRC was defined as the presence of liver metastases and limited lung (fewer than five nodules of $\leq 1 \mathrm{~cm}$ diameter or a single nodule of $\leq 1.7 \mathrm{~cm}$ diameter), and/or lymph node involvement (a single anatomic area of $<2 \mathrm{~cm}$ diameter). Bevacizumab was allowed, combined with mFOLFOX6, at the investigator's discretion (Fig 1A). ${ }^{16}$ The treatment schedules are described in Figure 1B.

Predefined stratification parameters included liver-only versus liver plus extrahepatic metastases (the aim was for at least $60 \%$ of recruited patients to have liver-only metastases), the extent of tumor involvement of the liver (classified as $\leq 25 \%$ or $>25 \%$ determined objectively on baseline computed tomography scan), intent to use bevacizumab with chemotherapy, and investigational center. ${ }^{16}$ All patients were monitored until death or for a maximum of 5 years.

\section{Outcome Measures}

The primary study end point was progression-free survival (PFS) at any site as assessed by independent centralized imaging review blinded to study arm. Secondary end points included PFS in the liver; tumor response rate in the liver; tumor response rate at any site; liver resection rate; hepatic and extrahepatic recurrence rate; health-related quality of life (analysis ongoing, not reported in this publication); toxicity and safety (adverse events [AEs] graded according to National Cancer Institute Common Toxicity Criteria version 3.0; serious AEs defined as any event resulting in death, that is lifethreatening, resulting in congenital anomaly, requiring or prolonging inpatient hospitalization, resulting in persistent or significant disability; or another medically important event); and OS, to be evaluated as a preplanned combined analysis of data from SIRFLOX and two other studies, FOXFIRE ${ }^{17}$ and FOXFIRE Global (clinicaltrials.gov identifier: NCT01721954).

The screening assessment was conducted $\leq 28$ days before random assignment. Patients were assessed subsequently on day 1 , day 3 or 4 , and every 2 weeks (plus or minus 1 week) during each chemotherapy cycle, and every 12 weeks after progression. ${ }^{16}$ Follow-up assessments included clinical assessment and physical examination, review of performance status, hematologic and biochemical assessments, serum carcinoembryonic antigen measurement, contrast-enhanced computed tomography of chest/abdomen/pelvis, assessment of suitability for liver resection, assessment of concurrent medications, and health-related quality of life assessment.

\section{Independent Blinded Evaluation of Radiologic Imaging}

The imaging response evaluation used response evaluation criteria in solid tumors (RECIST) version $1.0^{18}$ modified a priori as follows: (1) The documentation of tumor progression required an increase in the sum of the longest diameters of $\geq 20 \%$ and an absolute increase in the sum of the longest diameters of $\geq 5 \mathrm{~mm}$, or the appearance of a new lesion (analogous to the criteria from RECIST version 1.1). ${ }^{19}$ (2) Lymph nodes were assessed per RECIST version 1.1 (ie, a lymph node qualified as a potential target lesion when its short axis diameter was $\geq 15 \mathrm{~mm}$ at baseline).

All radiologic images were assessed in two separate and independent reading sessions by radiologists blinded to the study arm. In the event of discordance, a third independent radiologist adjudicated the image assessment to determine the final outcome.

The assessment of the pattern of progression included the site of progression; whether it was intra- or extrahepatic; and whether this occurred as a result of the growth of existing lesions, the appearance of new lesions, or both.

\section{Statistical Methods}

Using previously reported data on PFS with FOLFOX plus bevacizumab ${ }^{20}$ and on SIRT added to first-line fluoropyrimidine-based chemotherapy, ${ }^{14,21}$ a sample size of at least 450 patients for the SIRFLOX study was estimated to be needed to detect an increase in the median PFS at any site from 9.4 months to 12.5 months with $80 \%$ power and $95 \%$ confidence. Taking into account the number of patients who might receive the alternative treatment or lack imaging data, the sample size was increased to 530.

All efficacy measures were assessed in the intent-to-treat (ITT) population. Response rates were compared between treatment arms using a test of proportions, and time-to-event end points were compared using the log-rank test. A predefined competing risk analysis ${ }^{22}$ was used to assess PFS in the liver, to account for the competing risk of death or progression outside the liver. For unplanned exploratory analyses, $P<.01$ was used to define statistical significance.

\section{RESULTS}

Between October 2006 and April 2013, patients were recruited from 87 centers in Australia, Europe, Israel, New Zealand, and the United States. Of the 530 patients randomly assigned to treatment (ITT population), 263 were assigned to control and 267 were assigned to SIRT (Fig 2). There were no statistically significant differences between treatment arms in any characteristic at baseline (Table 1). 
A

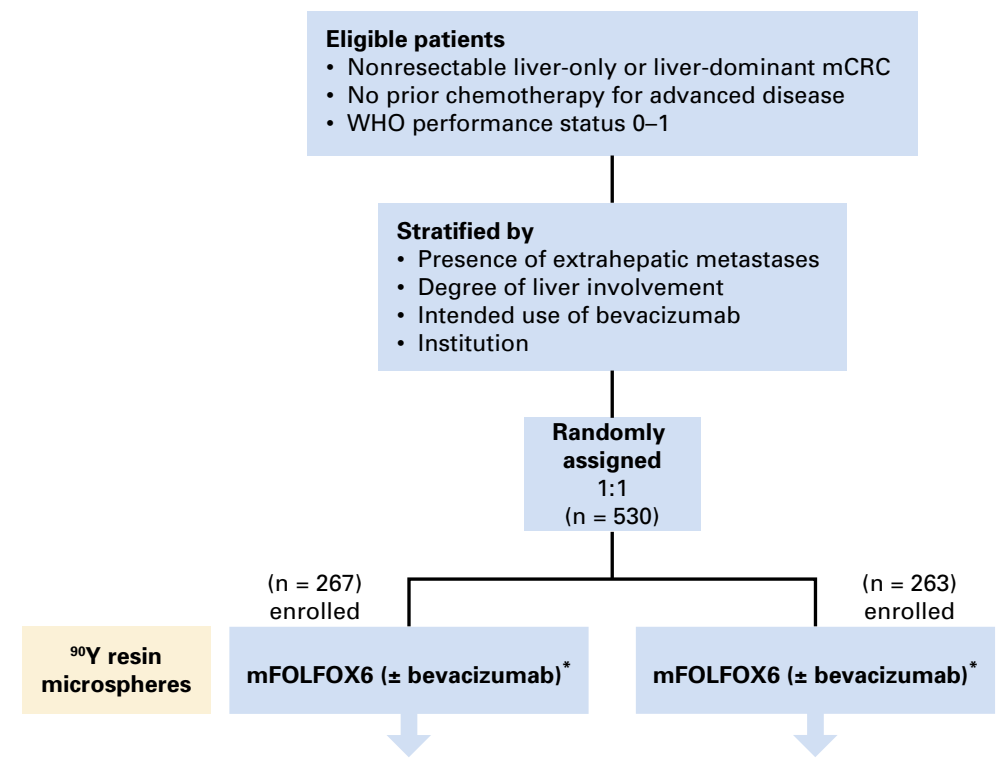

Primary end point: PFS in the ITT population by independent centralized imaging review

Secondary end points:

- PFS in the liver

- Tumor response rate in the liver

- Tumor response rate at any site (RECIST 1.0)

- Liver resection rate

- Hepatic and extrahepatic recurrence rate

- Toxicity and safety (NCI CTCAE v3.0)

- Health-related quality of life

- Overall survival (in a preplanned combined analysis)

B

Control arm: mFOLFOX6 ( \pm bevacizumab) ${ }^{*}$
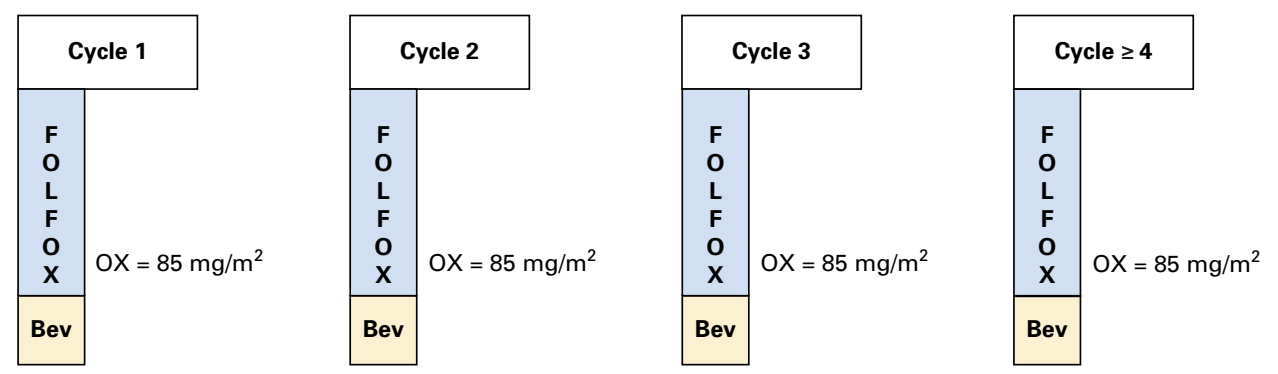

SIRT arm: mFOLFOX6 $( \pm \text { bevacizumab })^{*}+$ SIRT $\dagger$

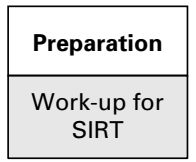

On day 14 to day 3

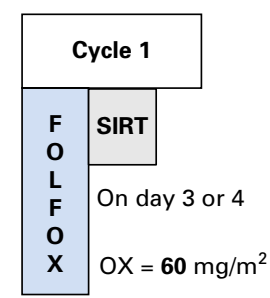

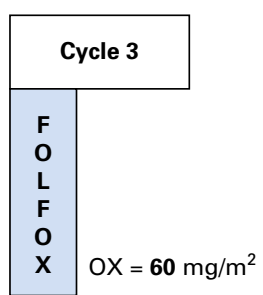

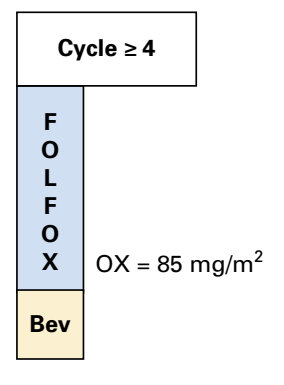

Fig 1. (A) Study design and end points. (B) Treatment schedules. *Bevacizumab allowed at investigator's discretion, per institutional practice; +Work-up procedure at day 14 to day 3 before SIRT; SIR-Spheres ${ }^{90} Y$ resin microspheres administered on either day 3 or day 4, of either cycle 1 or cycle 2. Bev, bevacizumab; IT, intent to treat; FOLFOX, fluorouracil, leucovorin, and oxaliplatin; mCRC, metastatic colorectal cancer; mFOLFOX6, modified fluorouracil, leucovorin, and oxaliplatin; NCI-CTCV3, National Cancer Institute Common Toxicity Criteria version 3; OX, oxaliplatin; PFS, progression-free survival; RECIST, Response Evaluation Criteria In Solid Tumors; SIRT, selective internal radiation therapy; ${ }^{90} Y$, yttrium-90. 


\section{Treatment}

The median (interquartile range [IQR]) number of cycles of fluorouracil administered was 12.0 (9.0) and 12.0 (9.0) in control and SIRT, respectively, and the median (IQR) number of cycles of oxaliplatin administered was 10.0 (4.0) and 10.0 (5.0) in control and SIRT, respectively. The median (IQR) number of cycles of bevacizumab administered was 13.0 (11.0) and 8.0 (8.0) in the 144 of 263 patients (54.8\%) and 125 of 267 patients (46.8\%) planned for bevacizumab treatment at study entry in control and SIRT, respectively, with bevacizumab administration in SIRT not to commence before cycle 4 .

In SIRT, ${ }^{90} \mathrm{Y}$ resin microspheres were implanted a median of 20 days after random assignment (range, 8 to 76 days), and the median implanted activity was 1.4 (range 0.4 to 3.1 ) GBq. Of the 21 patients in the SIRT arm who did not receive SIRT, 18 of 267 (7\%) were not able to receive SIRT and three of 267 (1\%) did not receive any study treatment as a consequence of compromised performance status, serious AEs, or disease progression before study treatment (Fig 2). Of the 246 patients who received SIRT, both liver lobes were treated in 227 patients $(92.3 \%)$ and a single lobe was treated in 19 (7.7\%). Of the 11 control patients $(4.2 \%)$ who did not receive any study treatment, 10 withdrew consent after randomization.

\section{Efficacy}

PFS at any site and PFS in the liver. Median PFS at any site was similar for control and SIRT (10.2 versus 10.7 months, respectively; hazard ratio $[\mathrm{HR}], 0.93$; 95\% $\mathrm{CI}, 0.77$ to $1.12 ; P=.43$; Fig 3 and Appendix Fig A1, online only). By competing risk analysis, the addition of SIRT improved median PFS in the liver from 12.6 (control) to 20.5 months (SIRT; HR, 0.69; $95 \% \mathrm{CI}, 0.55$ to 0.90 ; $P=.002$; Fig 4). This finding was consistent irrespective of tumor burden, bevacizumab therapy, or performance status (Appendix Fig A1).

Site of first disease progression. The numbers of patients with disease progression as their first study event in control and SIRT were 178 and 166, respectively (Table 2). First progression only in the liver occurred in a higher proportion of control versus SIRT patients $(77 \%$ versus 52.4\%; $P<.001$ ). There was a corresponding increase in first progression occurring outside the liver, particularly in the lung, for SIRT patients $(P<.001)$, but there was no significant difference in timing of lung progression compared with control patients (median time to lungonly progression events, 8.9 [control] $v 12.5$ [SIRT] months; $P=.049$ exploratory analysis). A higher proportion of first progression events occurred in existing lesions within the liver (plus or minus other sites) in control versus SIRT patients $(P<.001$; Appendix Table A2, online only).

ORR. The ORR at any site according to RECIST version 1.0 was not significantly different between control and SIRT (68.1\% v 76.4\%; $P=.113)$. In the liver, the ORR $(68.8 \% v 78.7 \% ; P=.042)$ and the complete response rate $(1.9 \% \vee 6.0 \% ; P=.020)$ were significantly improved with the addition of SIRT (Appendix Table A3, online only).

There was no significant difference between study arms in the rate of liver resection, with 36 patients (13.7\%) undergoing liver resection in control compared with 38 patients $(14.2 \%)$ in SIRT

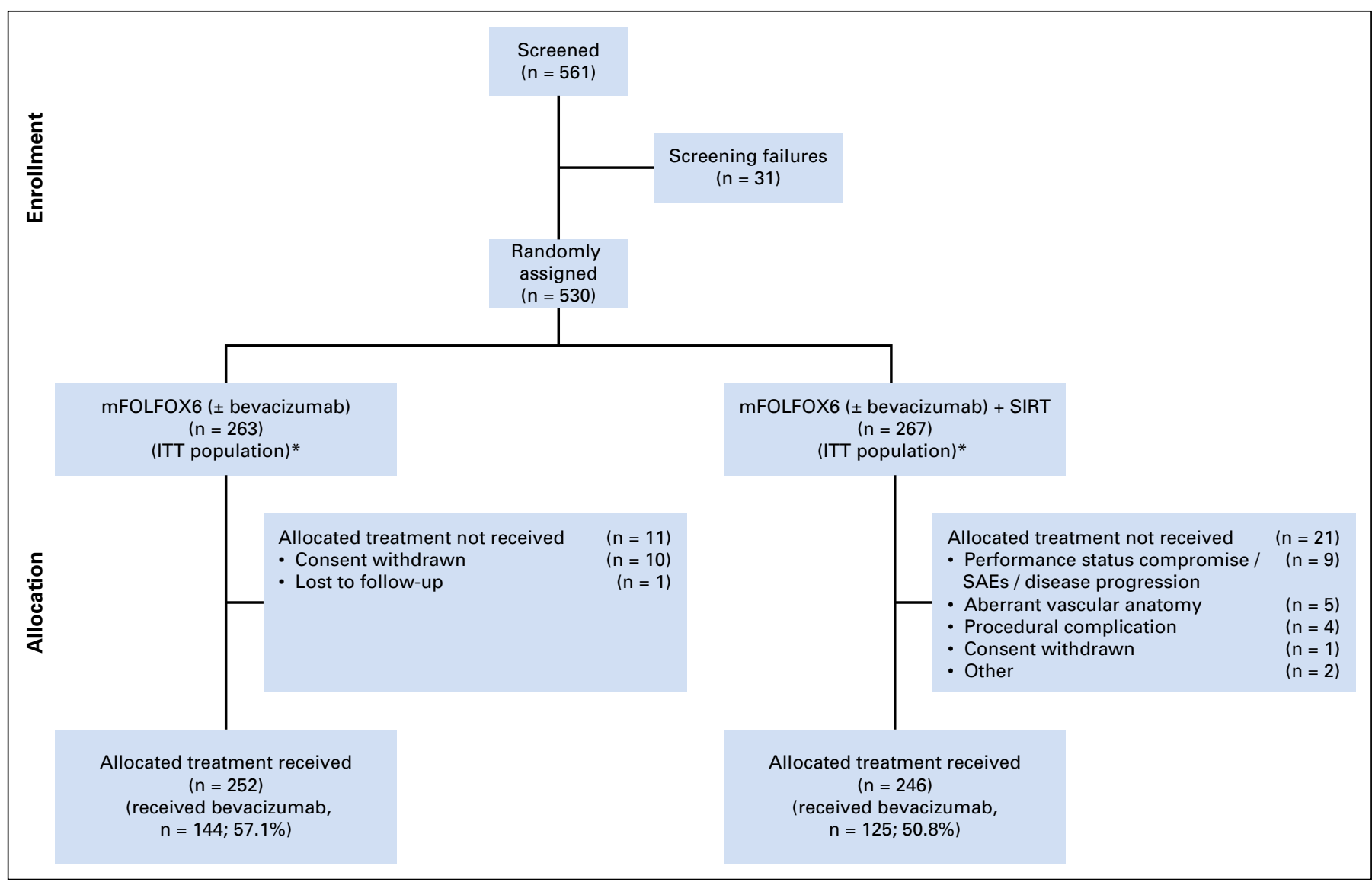

Fig 2. Patient disposition. *Included in primary outcome analysis. ITT, intent to treat; mFOLFOX6, modified fluorouracil, leucovorin, and oxaliplatin; SAE, serious adverse event; SIRT, selective internal radiation therapy. 
Table 1. Baseline Patient and Disease Characteristics (ITT Population)

\begin{tabular}{|c|c|c|}
\hline Variable & $\begin{array}{c}\text { Control Arm (mFOLFOX6 } \\
{[ \pm \text { bev]) } n=263}\end{array}$ & $\begin{array}{c}\text { Treatment Arm (SIRT }+ \text { mFOLFOX6 } \\
[ \pm \text { bev }]) n=267\end{array}$ \\
\hline Age, years, median (range) & $63(23-89)$ & $63(28-81)$ \\
\hline \multicolumn{3}{|l|}{ Sex } \\
\hline Male & $174(66.2)^{*}$ & $182(68.2)$ \\
\hline Female & $88(33.5)$ & $85(31.8)$ \\
\hline \multicolumn{3}{|l|}{ Race } \\
\hline White & $243(92.4)$ & 248 (92.9) \\
\hline Black & $8(3.0)$ & $2(0.7)$ \\
\hline Other & $7(2.7)$ & $11(4.1)$ \\
\hline Unknown & $5(1.9)$ & $6(2.2)$ \\
\hline \multicolumn{3}{|l|}{ WHO performance status } \\
\hline 0 & $175(66.5)$ & $176(65.9)$ \\
\hline 1 & $87(33.1)^{*}$ & $90(33.7)^{*}$ \\
\hline Extrahepatic metastases at randomization & 104 (39.5) & $108(40.4)$ \\
\hline Lungs alone & 36 (34.6) & $41(38.0)$ \\
\hline Lungs and lymph nodes & $18(17.3)$ & $14(13.0)$ \\
\hline Lymph nodes alone & $48(46.2)$ & $47(43.5)$ \\
\hline Unspecified & $2(1.9)$ & $6(5.6)$ \\
\hline Primary tumor in situ & $121(46.0)^{*}$ & 119 (44.6) \\
\hline \multicolumn{3}{|l|}{ Primary tumor location } \\
\hline Left & $137(52.1)$ & $141(52.8)$ \\
\hline Right & $55(20.1)$ & $72(27.0)$ \\
\hline Rectal & $59(22.4)$ & $45(16.9)$ \\
\hline Left + right & $4(1.5)$ & $5(1.9)$ \\
\hline Left + rectal & $4(1.5)$ & $2(0.7)$ \\
\hline Left + right + rectal & $2(0.8)$ & $0(0.0)$ \\
\hline Unknown & $2(0.8)$ & $2(0.7)$ \\
\hline Synchronous metastases & $233(88.6)$ & $241(90.3)$ \\
\hline \multicolumn{3}{|l|}{ Tumor liver involvement, \% } \\
\hline$\leq 25$ & $192(73.0)$ & $185(69.3)$ \\
\hline$>25$ & $70(26.6)^{*}$ & $81(30.3)^{*}$ \\
\hline Prior adjuvant chemotherapy & $16(6.1)$ & $13(4.9)$ \\
\hline Prior radiotherapy to nonliver sites & $14(5.3)$ & $11(4.1)$ \\
\hline \multicolumn{3}{|l|}{ Reasons for unresectability of liver metastases } \\
\hline Extrahepatic disease & $28(10.6)$ & $33(12.4)$ \\
\hline Insufficient liver reserve & $19(7.2)$ & $21(7.9)$ \\
\hline Proximity to major vessels & $14(5.3)$ & $13(4.9)$ \\
\hline Medically inoperable & $32(12.2)$ & $46(17.2)$ \\
\hline Patient age & $7(2.7)$ & $3(1.1)$ \\
\hline Tumor too large & $43(16.3)$ & $49(18.4)$ \\
\hline Too many tumors & $199(75.7)$ & $197(73.8)$ \\
\hline Attachment to another major structure & $1(0.4)$ & $1(0.4)$ \\
\hline Other & $9(3.4)$ & $10(3.7)$ \\
\hline
\end{tabular}

NOTE. Data are presented as No. (\%) unless indicated otherwise.

Abbreviations: bev, bevacizumab; ITT, intent to treat; mFOLFOX6, modified fluorouracil, leucovorin, and oxaliplatin; SIRT, selective internal radiation therapy.

*Unknown for one patient: patient withdrew consent and records.

$(P=.857)$; all patients had been considered unresectable at study entry by a multidisciplinary team.

\section{Safety}

Treatment-emergent grade $\geq 3$ AEs were reported in $73.3 \%$ of control and $85.4 \%$ of SIRT patients (Table 3). Hematologic toxicities were reported at a higher rate in SIRT compared with control $(P<.05)$. Known SIRT-associated AEs were reported in SIRT patients only (Table 3 ). Eight of the nine grade $\geq 3$ gastric or duodenal ulcers were considered SIRT related, and one patient $(0.4 \%)$ developed a grade 4 ulcer. Two patients with ulcers required surgical management.

Grade $5 \mathrm{AEs}$ of any causality were reported in five patients $(1.9 \%)$ in control and nine patients $(3.7 \%)$ in SIRT $(P=.279)$. Four treatment-related grade 5 AEs were attributed to chemotherapy (two cardiac-related events in control, and one respiratory failure and one febrile neutropenia in SIRT), two were attributed to SIRT (hepatic failure and radiation hepatitis), and one was attributed to both chemotherapy and SIRT (hepatic failure in SIRT).

Serious AEs were reported less frequently in control patients $(41.6 \%)$ than in SIRT patients $(54.1 \% ; P=.005)$. AEs that led to reduction, delay, or discontinuation of protocol therapy occurred in $9.3 \%, 33.1 \%$, and $15.6 \%$ of control and $4.1 \%, 41.5 \%$, and $17.5 \%$ of SIRT patients, respectively.

Five patients with SIRT-related hepatotoxicity (radiation hepatitis or hepatic failure) were managed with supportive treatment. Both cases of radiation hepatitis, one of which was fatal, occurred 2 to 3 months after SIRT and were treated with low-molecular-weight heparin, diuretics, and corticosteroids. Two patients experienced fatal hepatic failure, one case occurring 5 days after SIRT and the 


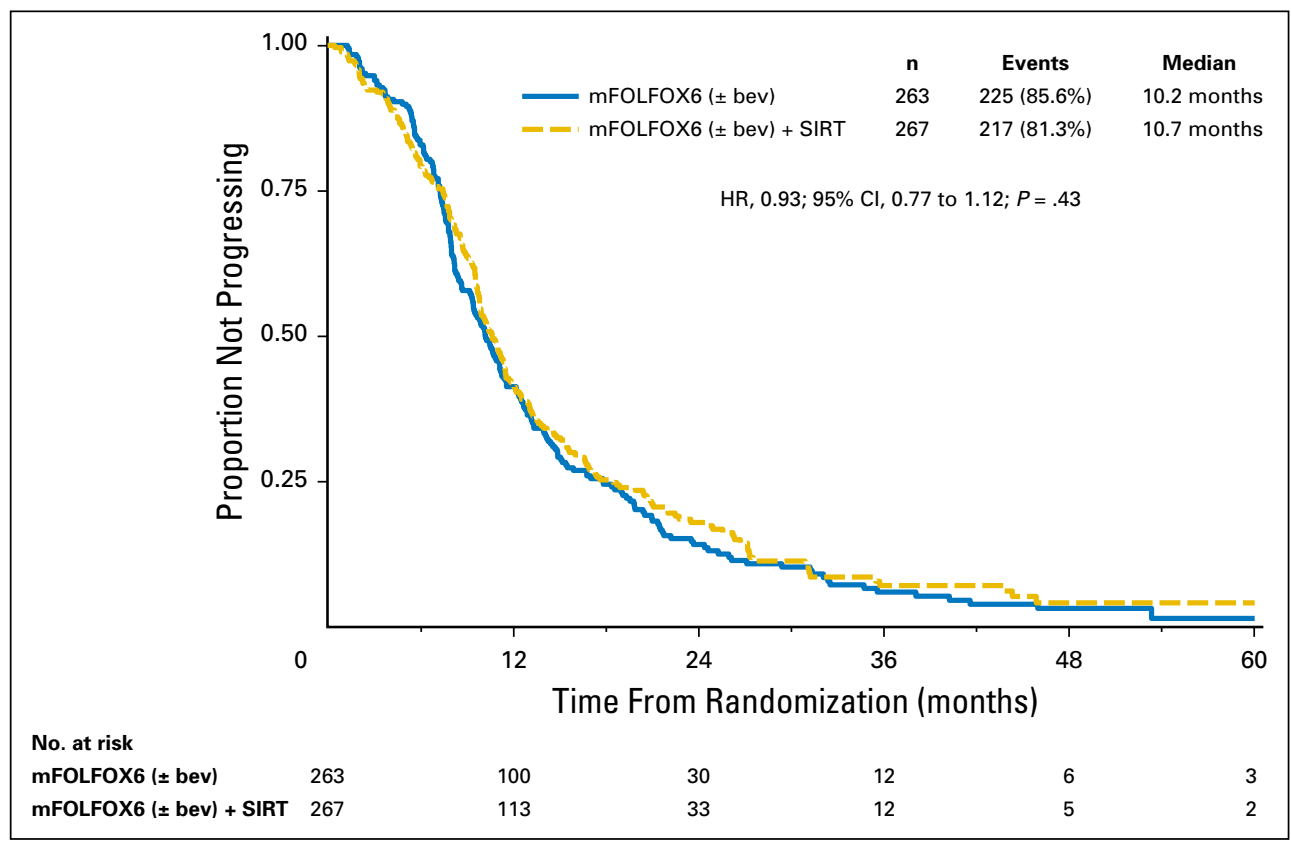

Fig 3. Kaplan-Meier analysis of progressionfree survival at any site (intent-to-treat population) determined by independent centralized imaging review. bev, bevacizumab; HR, hazard ratio; mFOLFOX6, modified fluorouracil, leucovorin, and oxaliplatin; SIRT, selective internal radiation therapy.

other case $>2$ years after SIRT. The latter case presented with portal hypertension (splenomegaly and thrombocytopenia) and subsequently developed hepatic insufficiency consistent with prior subclinical radiation hepatitis. ${ }^{23}$ A third (nonfatal) case of hepatic failure in a SIRT patient occurred 1 day after resection of liver metastases.

\section{DISCUSSION}

SIRFLOX is the first large phase III randomized controlled trial to assess the efficacy and safety of a liver-directed therapy in patients with mCRC. Unlike previous studies combining SIRT with firstline fluoropyrimidine-based chemotherapy in patients with mCRC, the SIRFLOX study failed to show an improvement in median PFS at any site with the addition of SIRT. The addition of SIRT significantly improved median PFS in the liver by 7.9 months, corresponding to a $31 \%$ risk reduction. OS data from a combined analysis of SIRFLOX and two other first-line studies are awaited to determine whether this substantial gain in control of existing liver metastases translates into a significant gain in survival.

The site and pattern of first disease progression in control and SIRT patients offer insight into the apparent discordance between

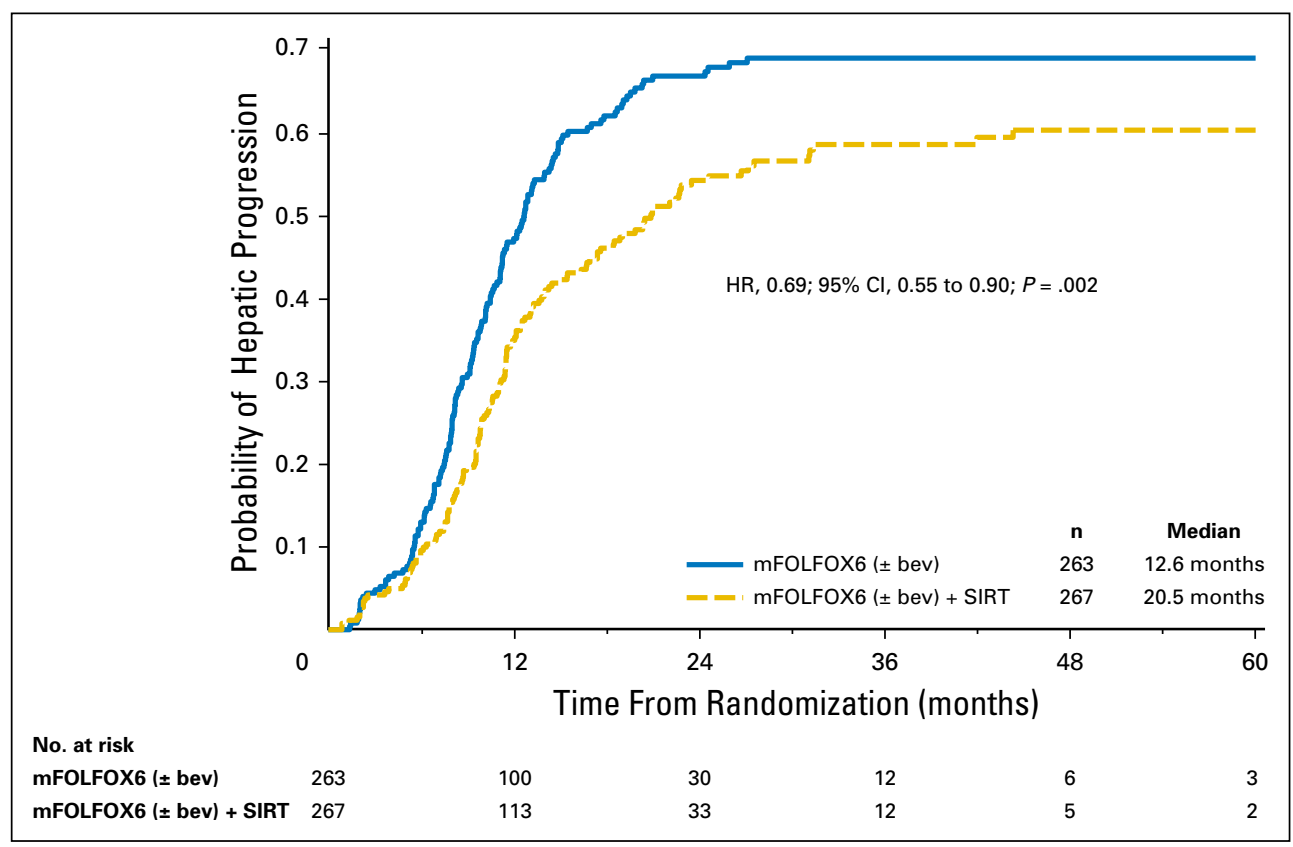

Fig 4. Cumulative incidence of liver progression (intent-to-treat population) determined by independent central image reading, accounting for the risk of death or progression outside the liver. bev, bevacizumab; HR, hazard ratio; mFOLFOX6, modified fluorouracil, leucovorin, and oxaliplatin; SIRT, selective internal radiation therapy. 


\begin{tabular}{|c|c|c|c|}
\hline Number of First Disease Progression and Site(s) & Control Arm (mFOLFOX6 [ \pm bev]) & Treatment Arm (SIRT + mFOLFOX6 [ \pm bev]) & $P$ \\
\hline Number of first progressions & 178 & 166 & \\
\hline \multicolumn{4}{|l|}{ Site(s) of first disease progression } \\
\hline Liver only & $137(77.0)$ & $87(52.4)$ & $<.001$ \\
\hline Liver + nonliver sites & $27(15.2)$ & 33 (19.9) & .251 \\
\hline Liver + lung & $21(11.8)$ & 29 (17.5) & .136 \\
\hline Liver + lymph nodes & $6(3.4)$ & $2(1.2)$ & .184 \\
\hline Liver + lung + lymph nodes & $0(0.0)$ & $1(0.6)$ & .300 \\
\hline Liver + abdominal wall & $0(0.0)$ & $1(0.6)$ & .300 \\
\hline Nonliver sites only & $14(7.9)$ & $46(27.7)$ & $<.001$ \\
\hline Lung only & $13(7.3)$ & $39(23.5)$ & $<.001$ \\
\hline Lymph nodes only & $1(0.6)$ & $5(3.0)$ & .083 \\
\hline Lung + lymph nodes & $0(0.0)$ & $2(1.2)$ & .142 \\
\hline
\end{tabular}

NOTE. Data are presented as No. (\%) unless indicated otherwise.

Abbreviations: bev, bevacizumab; mFOLFOX6, modified fluorouracil, leucovorin, and oxaliplatin; SIRT, selective internal radiation therapy.

PFS at any site and PFS in the liver. Whereas control of intra- and extrahepatic disease is required to achieve a benefit in PFS at any site, the analysis of first progression site suggests that progressive disease in nonliver sites $(7.9 \% v 27.7 \%)$ may mitigate the benefit of controlling liver disease with SIRT. Furthermore, the appearance of new lesions accounted for a substantially greater proportion of first progressions in the liver in SIRT (Appendix Table A2). Collectively, these data suggest that although SIRT used in conjunction with systemic chemotherapy provides prolonged control of evident liver disease, this is insufficient to influence PFS at any site. The increased incidence of progression within the lungs for SIRT patients would seem to reflect lung progression destined to occur in patients receiving a liver-directed intervention.

Other factors that may have compromised the ability of SIRT to significantly affect PFS at any site and the gains achieved in control of liver metastases include the 21 patients $(7.9 \%)$ randomly assigned to SIRT but not receiving SIRT and the 19 patients $(7.7 \%)$ with bilobar disease who received SIRT in only one liver lobe. Ultimately, only $84 \%$ of patients allocated to SIRT received SIRT as per protocol. This is explained partly by the random assignment of patients before consideration of their suitability for SIRT, but, on the basis of previous experience, the proportion of patients not receiving SIRT as per protocol was unexpectedly high. ${ }^{14,15,24}$ Also unanticipated was the large proportion of patients (approximately 45\%) with an intact primary tumor; this had an uncertain impact on the primary study end point of PFS at any site and was reported to be associated with inferior survival outcomes. ${ }^{25}$ There are also uncertainties regarding the 10 patients $(3.8 \%)$ who withdrew consent after being randomly assigned to control (and may have received SIRT off protocol as part

\begin{tabular}{|c|c|c|c|}
\hline Adverse Event & Control Arm (mFOLFOX6 [ \pm bev]) & Treatment Arm (SIRT + mFOLFOX6 [ \pm bev $])$ & $P$ \\
\hline Safety population & $270(100)$ & $246(100)$ & \\
\hline Total $\geq$ grade $3 \mathrm{AEs}$ & 198 (73.3) & $210(85.4)$ & .516 \\
\hline \multicolumn{4}{|l|}{ Any* } \\
\hline Neutropenia & 77 (28.5) & $100(40.7) \dagger$ & .004 \\
\hline Febrile neutropenia & $5(1.9)$ & $15(6.1) \dagger$ & .020 \\
\hline Thrombocytopenia & $7(2.6)$ & $24(9.8) \dagger$ & $<.001$ \\
\hline Diarrhea & $24(8.9)$ & 18 (7.3) & .535 \\
\hline Peripheral neuropathy & $23(8.5)$ & $14(5.7)$ & .235 \\
\hline Pulmonary embolism & $15(5.6)$ & $17(6.9)$ & .586 \\
\hline Fatigue & $13(4.8)$ & $26(10.6) \dagger$ & .019 \\
\hline Nausea/vomiting & $11(4.1)$ & $20(8.1)$ & .064 \\
\hline Abdominal pain & $7(2.6)$ & $19(7.7) \dagger$ & .009 \\
\hline \multicolumn{4}{|l|}{ SIRT-associated eventsł } \\
\hline Gastric/duodenal ulcer & $0(0.0)$ & $9(3.7) \dagger$ & .001 \\
\hline Ascites & $0(0.0)$ & $7(2.8) \dagger$ & .005 \\
\hline Hepatic failure & $0(0.0)$ & $3(1.2)$ & .108 \\
\hline Radiation hepatitis & $0(0.0)$ & $2(0.8)$ & .227 \\
\hline Total grade 5 AEs§ & $5(1.9)$ & $9(3.7)$ & .279 \\
\hline Treatment-related grade $5 \mathrm{AEs} \|$ & $2(0.7)$ & $5(2.0)$ & .266 \\
\hline \multicolumn{4}{|c|}{$\begin{array}{l}\text { NOTE. Data are presented as No. (\%). } \\
\text { Abbreviations: AE, adverse event; bev, bevacizumab; mFOLFOX6, modified fluorouracil, leucovorin, and oxaliplatin; SIRT, selective internal radiation therapy. } \\
\text { *All grade } \geq 3 \text { adverse events occurring with an incidence of } \geq 5 \% \text { in either study arm, irrespective of attribution to treatment. } \\
\text { tStatistically significant difference in incidence }(P<.05) \text {. } \\
\text { †AEs typically associated with SIRT. } \\
\text { §Occurring with an incidence of }>0 \% \text { in either study arm, irrespective of attribution to treatment. } \\
\| \text { Attributed to either or both treatments. }\end{array}$} \\
\hline
\end{tabular}


of first-line therapy) but were included in the ITT population PFS analyses for control.

The median 20.5-month liver PFS for patients treated with chemotherapy plus SIRT represents a substantial prolongation of local disease control compared with systemic chemotherapy alone (median, 12.6 months). Because, to the best of our knowledge, this is the first study to evaluate PFS in the liver, there are no other studies that provide context for this result. However, recently reported data from the Chemotherapy + Local Ablation Versus Chemotherapy (CLOCC) study, which combined radiofrequency ablation with FOLFOX-based systemic chemotherapy in patients with unresectable mCRC confined to the liver, demonstrated that improved control of hepatic metastases can translate to a substantial impact on OS. $^{26}$ In contrast to those in SIRFLOX, all patients randomly assigned in the CLOCC study, which also demonstrated an improvement in PFS at any site (HR, 0.57; 95\% CI 0.38 to $0.85 ; P=.005$ ), had a low burden of liver disease and no extrahepatic disease, and all had had their primary CRC resected.

OS is a secondary outcome for the SIRFLOX study. During the 7-year recruitment period of the study, when it became evident that improved patient care and new chemotherapy regimens were extending survival for patients with $\mathrm{mCRC}$ receiving first-line chemotherapy treatment, ${ }^{20,27-32}$ a decision was made to preplan a combined OS analysis including data from SIRFLOX and two additional randomized studies (Sharma et $\mathrm{al}^{17}$ and NCT NCT01721954). In all three studies, SIRT has been added to oxaliplatin-based chemotherapy in an almost identical patient population. The FOXFIRE and FOXFIRE Global studies have completed accrual and, combined with SIRFLOX, have a total recruitment of $>1,100$ patients; this provides adequate power to detect a survival advantage. The result of the combined OS analysis is anticipated in 2017.

Despite the addition of SIRT improving ORR in the liver, there was no difference in liver resection rates in the two study arms, in contrast to previous studies in which increasing response rates have typically translated into increased liver resection rates. ${ }^{33}$ At study entry, the dominant reason for metastases not being resectable as recorded in the case report form was the number of liver metastases, suggesting that liver disease would not become resectable irrespective of response. For the $40 \%$ of patients with extrahepatic disease, an improvement in liver response rates is also unlikely to affect resection rates because liver resection is generally pursued only when all sites of disease can be resected completely. Of uncertain significance is the potential reluctance of liver surgeons to operate after SIRT even though there are no reliable data to suggest that surgical outcomes or complications are worse after SIRT.

The combination of SIRT with mFOLFOX6 resulted in a predictable increase in grade $\geq 3$ AEs attributable to both chemotherapy and SIRT. Common grade $\geq 3$ AEs associated with chemotherapy such as neutropenia, febrile neutropenia, and thrombocytopenia occurred more frequently with the addition of SIRT (28.5\% $v 40.7 \%, 1.9 \% v 6.1 \%$, and $2.6 \% v 9.7 \%$, respectively). The frequency of events reported in this study is consistent with reports of other studies with mFOLFOX6 plus or minus bevacizumab-based regimens (grade $\geq 3$ neutropenia, 44\%-53\%; grade $\geq 3$ thrombocytopenia , $5 \%-6 \%) .{ }^{34,35}$ There was an increase in grade $\geq 3$ toxicities known to be associated with SIRT caused by the acute effects of radiation (eg, nausea, vomiting, abdominal pain, and fatigue), nontarget implantation (gastric or duodenal ulcers), and hepatotoxicity (ascites, radiation hepatitis, and hepatic failure). SIRT-related toxicities were predictable and were predominantly medically manageable (but two patients required surgical intervention for duodenal or gastric ulcers). No previously unreported toxicities emerged.

In conclusion, the addition of SIRT, using ${ }^{90} \mathrm{Y}$ resin microspheres, to standard FOLFOX-based first-line systemic chemotherapy in patients with liver-dominant mCRC did not improve PFS at any site but significantly delayed progression in the liver. The addition of SIRT did not adversely affect the delivery of chemotherapy, and the AE profile was anticipated and manageable. No unexpected toxicities were observed. The potential long-term impact on survival from integrating SIRT into the first-line treatment of mCRC will be evident when the results of the preplanned combined analysis of SIRFLOX, FOXFIRE, and FOXFIRE Global are available.

\section{AUTHORS' DISCLOSURES OF POTENTIAL CONFLICTS} OF INTEREST

Disclosures provided by the authors are available with this article at www.jco.org.

\section{AUTHOR CONTRIBUTIONS}

Conception and design: Guy A. van Hazel, David N. Cade, Peter Gibbs Provision of study materials or patients: Guy A. van Hazel, Jens Ricke, Bridget A. Robinson, Tom Ferguson, Eveline Boucher, Julien Taieb, Peter Gibbs

Collection and assembly of data: Guy A. van Hazel, Navesh K. Sharma, Michael P.N. Findlay, Bridget A. Robinson, Andrew H. Strickland, Tom Ferguson, Javier Rodríguez, Ido Wolf, Vinod Ganju, Eveline Boucher, Einat Shacham-Shmueli, Alex Powell, Paul Eliadis, Richard Isaacs, David Price, Fred Moeslein, Julien Taieb, Geoff Bower

Data analysis and interpretation: Guy A. van Hazel, Volker Heinemann, Michael P.N. Findlay, Jens Ricke, Marc Peeters, David Perez, Andrew H. Strickland, Hendrik Kröning, Euan Walpole, Thomas Tichler, Val Gebski, Mark Van Buskirk, Kenneth Thurston, Peter Gibbs

Manuscript writing: All authors

Final approval of manuscript: All authors

\section{REFERENCES}

1. Torre LA, Bray F, Siegel RL, et al: Global cancer statistics, 2012. CA Cancer J Clin 65:87-108, 2015

2. Berber E, Pelley R, Siperstein AE: Predictors of survival after radiofrequency thermal ablation of colorectal cancer metastases to the liver: A prospective study. J Clin Oncol 23:1358-1364, 2005

3. Navarra G, Ayav A, Weber JC, et al: Short-andlong term results of intraoperative radiofrequency ablation of liver metastases. Int J Colorectal Dis 20: 521-528, 2005
4. Rothbarth J, van de Velde CJ: Treatment of liver metastases of colorectal cancer. Ann Oncol 16 (Suppl 2):ii144-ii149, 2005 [Erratum Ann Oncol17: 727, 2006]

5. Van Cutsem E, Nordlinger B, Adam R, et al; European Colorectal Metastases Treatment Group: Towards a pan-European consensus on the treatment 
of patients with colorectal liver metastases. Eur J Cancer 42:2212-2221, 2006

6. Abbas S, Lam V, Hollands M: Ten-year survival after liver resection for colorectal metastases: Systematic review and meta-analysis. ISRN Oncol 2011: 763245, 2011

7. Adam R: Developing strategies for liver metastases from colorectal cancer. Semin Oncol 34(2, Suppl 1)S7-S11, 2007

8. Welch JP, Donaldson GA: The clinical correlation of an autopsy study of recurrent colorectal cancer. Ann Surg 189:496-502, 1979

9. Helling TS, Martin M: Cause of death from liver metastases in colorectal cancer. Ann Surg Oncol 21: 501-506, 2014

10. Kennedy A, Nag S, Salem R, et al: Recommendations for radioembolization of hepatic malignancies using yttrium-90 microsphere brachytherapy: A consensus panel report from the radioembolization brachytherapy oncology consortium. Int J Radiat Oncol Biol Phys 68:13-23, 2007

11. Sangro B, Inarrairaegui M: Radioembolization for hepatocellular carcinoma: Evidence-based answers to frequently asked questions. J Nucl Med Radiat Ther 2:1000110, 2011

12. Gulec SA, Pennington $K$, Hall $M$, et al: Preoperative $\mathrm{Y}-90$ microsphere selective internal radiation treatment for tumor downsizing and future liver remnant recruitment: A novel approach to improving the safety of major hepatic resections. World J Surg Oncol 7:6, 2009

13. Ricke J, Bulla K, Kolligs $F$, et al; SORAMIC study group: Safety and toxicity of radioembolization plus Sorafenib in advanced hepatocellular carcinoma: Analysis of the European multicentre trial SORAMIC. Liver Int 35:620-626, 2015

14. Van Hazel G, Blackwell A, Anderson J, et al: Randomised phase 2 trial of SIR-Spheres plus fluorouracil/ leucovorin chemotherapy versus fluorouracil/leucovorin chemotherapy alone in advanced colorectal cancer. J Surg Oncol 88:78-85, 2004

15. Sharma RA, Van Hazel GA, Morgan B, et al: Radioembolization of liver metastases from colorectal cancer using yttrium-90 microspheres with concomitant systemic oxaliplatin, fluorouracil, and leucovorin chemotherapy. J Clin Oncol 25:1099-1106, 2007

16. Gibbs P, Gebski V, Van Buskirk M, et al; SIRFLOX Study Group: Selective internal radiation therapy (SIRT) with yttrium-90 resin microspheres plus standard systemic chemotherapy regimen of FOLFOX versus FOLFOX alone as first-line treatment of non-resectable liver metastases from colorectal cancer: The SIRFLOX study. BMC Cancer 14:897, 2014

17. Sharma RA, Wasan HS, Love $S B$, et al; FOXFIRE Trial Management Group: FOXFIRE: A phase III clinical trial of chemo-radio-embolisation as first-line treatment of liver metastases in patients with colorectal cancer. Clin Oncol (R Coll Radiol) 20: 261-263, 2008

18. Therasse $P$, Arbuck SG, Eisenhauer EA, et al: New guidelines to evaluate the response to treatment in solid tumors. European Organization for Research and Treatment of Cancer, National Cancer Institute of the United States, National Cancer Institute of Canada. J Natl Cancer Inst 92:205-216, 2000

19. Eisenhauer EA, Therasse $P$, Bogaerts J, et al: New response evaluation criteria in solid tumours: Revised RECIST guideline (version 1.1). Eur J Cancer 45:228-247, 2009

20. Saltz LB, Clarke S, Díaz-Rubio E, et al: Bevacizumab in combination with oxaliplatin-based chemotherapy as first-line therapy in metastatic colorectal cancer: A randomized phase III study. J Clin Oncol 26: 2013-2019, 2008

21. Gray B, Van Hazel G, Hope M, et al: Randomised trial of SIR-Spheres plus chemotherapy vs. chemotherapy alone for treating patients with liver metastases from primary large bowel cancer. Ann Oncol 12:1711-1720, 2001

22. Gray RJ: A class of K-sample tests for comparing the cumulative incidence of a competing risk. Ann Stat 16:1141-1154, 1988

23. Stubbs RS, Wickremesekera SK: Selective internal radiation therapy (SIRT): A new modality for treating patients with colorectal liver metastases. HPB (Oxford) 6:133-139, 2004

24. van Hazel GA, Pavlakis N, Goldstein D, et al: Treatment of fluorouracil-refractory patients with liver metastases from colorectal cancer by using yttrium90 resin microspheres plus concomitant systemic irinotecan chemotherapy. J Clin Oncol 27:4089-4095, 2009

25. Faron M, Pignon JP, Malka D, et al: Is primary tumour resection associated with survival improvement in patients with colorectal cancer and unresectable synchronous metastases? A pooled analysis of individual data from four randomised trials. Eur J Cancer 51:166-176, 2015

26. Ruers T, Punt CJA, Van Coevorden F, et al: Radiofrequency ablation (RFA) combined with chemotherapy for unresectable colorectal liver metastases (CRC LM): Long-term survival results of a randomized phase II study of the EORTC-NCRI CCSG-ALM Intergroup 40004 (CLOCC). J Clin Oncol 33:A3501, 2015

27. Saltz LB, Cox JV, Blanke C, et al; Irinotecan Study Group: Irinotecan plus fluorouracil and leucovorin for metastatic colorectal cancer. N Engl J Med 343:905-914, 2000

28. Falcone A, Ricci S, Brunetti I, et al; Gruppo Oncologico Nord Ovest: Phase III trial of infusional fluorouracil, leucovorin, oxaliplatin, and irinotecan (FOLFOXIRI) compared with infusional fluorouracil, leucovorin, and irinotecan (FOLFIRI) as first-line treatment for metastatic colorectal cancer: the Gruppo Oncologico Nord Ovest. J Clin Oncol 25: 1670-1676, 2007

29. Van Cutsem $\mathrm{E}$, Köhne $\mathrm{CH}$, Láng $\mathrm{I}$, et al: Cetuximab plus irinotecan, fluorouracil, and leucovorin as first-line treatment for metastatic colorectal cancer: Updated analysis of overall survival according to tumor KRAS and BRAF mutation status. J Clin Oncol 29:2011-2019, 2011

30. Douillard JY, Siena S, Cassidy J, et al: Randomized, phase III trial of panitumumab with infusional fluorouracil, leucovorin, and oxaliplatin (FOLFOX4) versus FOLFOX4 alone as first-line treatment in patients with previously untreated metastatic colorectal cancer: The PRIME study. J Clin Oncol 28:4697-4705, 2010

31. Douillard JY, Oliner KS, Siena $S$, et al: Panitumumab-FOLFOX4 treatment and RAS mutations in colorectal cancer. N Engl J Med 369: 1023-1034, 2013

32. Van Cutsem E, Lenz $\mathrm{HJ}$, Köhne $\mathrm{CH}$, et al: Fluorouracil, leucovorin, and irinotecan plus cetuximab treatment and RAS mutations in colorectal cancer. J Clin Oncol 33:692-700, 2015

33. Folprecht G, Grothey A, Alberts $S$, et al: Neoadjuvant treatment of unresectable colorectal liver metastases: Correlation between tumour response and resection rates. Ann Oncol 16:1311-1319, 2005

34. Tournigand $C$, André T, Achille E, et al: FOLFIRI followed by FOLFOX6 or the reverse sequence in advanced colorectal cancer: A randomized GERCOR study. J Clin Oncol 22:229-237, 2004

35. Hochster HS, Hart LL, Ramanathan RK, et al: Safety and efficacy of oxaliplatin and fluoropyrimidine regimens with or without bevacizumab as first-line treatment of metastatic colorectal cancer: Results of the TREE Study. J Clin Oncol 26: 3523-3529, 2008

\section{Affiliations}

Guy A. van Hazel, University of Western Australia; Tom Ferguson, Royal Perth Hospital; David Price and Geoff Bower, Mount Medical Center, Perth; Alex Powell, Hollywood Private Hospital, Nedlands, Western Australia; Andrew H. Strickland, Monash Medical Centre, Bentleigh, East Victoria; Vinod Ganju, Frankston Private Hospital Peninsula Oncology Centre, Frankston; Peter Gibbs, Western Hospital, Footscray, Victoria; Euan Walpole, Princess Alexandra Hospital, Woolloongabba; Paul Eliadis, Wesley Medical Centre, Milton, Queensland; Val Gebski, NHMRC Clinical Trials Centre, Camperdown; David N. Cade and Kenneth Thurston, Sirtex Medical Limited, North Sydney, New South Wales, Australia; Volker Heinemann, Ludwig-Maximilian-University of Munich, Munich; Jens Ricke, University Clinic Magdeburg; Hendrik Kroening, Schwerpunktpraxis of Haematology and Oncology, Magdeburg, Germany; Navesh K. Sharma, University of Maryland Medical Center; Fred Moeslein, University of Maryland School of Medicine, Baltimore, MD; Mark Van Buskirk, Data Reduction LLC, Chester, NJ; Michael P.N. Findlay, Cancer Trials New Zealand, Auckland; David Perez, Dunedin Hospital, Dunedin; Bridget A. Robinson, Christchurch Hospital, Christchurch; Richard Isaacs, Palmerston North Hospital, Palmerston, New Zealand; Marc Peeters, Antwerp University Hospital, Antwerp, Belgium; Javier Rodríguez, Clinica Universidad de Navarra, Pamplona, Spain; Ido Wolf and Einat Shacham-Shmueli, Sheba Medical Center, Tel-Hashomer; Thomas Tichler, Shaare-Zedek Medical Center, Jerusalem, Israel; Eveline Boucher, Hopital de Jour, Rennes; and Julien Taieb, Georges Pompidou European Hospital, Paris, France. 
SIRFLOX: Randomized Phase III Trial Comparing First-Line mFOLFOX6 (Plus or Minus Bevacizumab) Versus mFOLFOX6 (Plus or Minus Bevacizumab) Plus Selective Internal Radiation Therapy in Patients with Metastatic Colorectal Cancer

The following represents disclosure information provided by authors of this manuscript. All relationships are considered compensated. Relationships are self-held unless noted. I = Immediate Family Member, Inst = My Institution. Relationships may not relate to the subject matter of this manuscript. For more information about ASCO's conflict of interest policy, please refer to www.asco.org/rwc or jco.ascopubs.org/site/ifc.

Guy A. van Hazel

Honoraria: Sirtex

Consulting or Advisory Role: Sirtex, Roche, Merck

Research Funding: Sirtex, Boehringer Ingelheim, Merck

Travel, Accommodations, Expenses: Sirtex, Boehringer Ingelheim

\section{Volker Heinemann}

Honoraria: Roche, Celgene, Amgen, Sanofi, Merck, Sirtex, Baxalta Consulting or Advisory Role: Merck, Amgen, Roche, Sanofi, Boehringer Ingelheim, Celgene, Sirtex, Baxalta

Research Funding: Merck, Amgen, Roche, Sanofi, Celgene, Boehringer Ingelheim, Sirtex

Travel, Accommodations, Expenses: Merck, Roche, Sirtex, Amgen,

Baxalta

Navesh K. Sharma

Honoraria: Sirtex

Speakers' Bureau: Sirtex

Michael P.N. Findlay

Consulting or Advisory Role: Sirtex

Travel, Accommodations, Expenses: Sirtex

Jens Ricke

Honoraria: Sirtex

Speakers' Bureau: Sirtex

Research Funding: Sirtex

Travel, Accommodations, Expenses: Sirtex

Marc Peeters

Honoraria: Sirtex

Consulting or Advisory Role: Sirtex

David Perez

Travel, Accommodations, Expenses: Roche

\section{Bridget A. Robinson}

Research Funding: Sirtex

Andrew H. Strickland

No relationship to disclose

Tom Ferguson

Travel, Accommodations, Expenses: Astellas Pharma

Javier Rodríguez

No relationship to disclose

\section{Hendrik Kröning}

No relationship to disclose

Ido Wolf

Research Funding: Ipsen, Novartis

Vinod Ganju

No relationship to disclose

\section{Euan Walpole}

Consulting or Advisory Role: Eli Lilly, Merck Serono

Travel, Accommodations, Expenses: Roche

Eveline Boucher

No relationship to disclose

\section{Thomas Tichler}

No relationship to disclose

Einat Shacham-Shmueli

No relationship to disclose

Alex Powell

No relationship to disclose

Paul Eliadis

No relationship to disclose

Richard Isaacs

No relationship to disclose

David Price

No relationship to disclose

Fred Moeslein

Honoraria: Sirtex

Travel, Accommodations, Expenses: Sirtex

Julien Taieb

Honoraria: Genentech, Merck Serono, Amgen, Celgene, Sanofi, Eli Lilly/ ImClone Systems

\section{Geoff Bower}

No relationship to disclose

Val Gebski

Consulting or Advisory Role: Sirtex

Travel, Accommodations, Expenses: Sirtex

Mark Van Buskirk

Consulting or Advisory Role: Sirtex

David N. Cade

Employment: Sirtex

Leadership: Sirtex

Stock or Other Ownership: Sirtex

Travel, Accommodations, Expenses: Sirtex

Kenneth Thurston

Employment: Sirtex

Leadership: Sirtex

Stock or Other Ownership: Sirtex

Peter Gibbs

Honoraria: Roche, Amgen, Merck, Sirtex, Alchemia

Research Funding: Roche 


\section{Acknowledgment}

We thank all investigators in the SIRFLOX Study Group, and in particular the principal investigators. We acknowledge the editorial assistance provided by Martin Gilmour of ESP Bioscience (Crowthorne, United Kingdom) funded by Sirtex, during the preparation of this article.

\section{Appendix}

\section{Principal Investigators}

Australia: Michael Brown, Matthew Burge, Giuseppe Cardaci, Stephen Clarke, Paul Eliadis, Tom Ferguson, Vinod Ganju, Philip James, Winston Liauw, Gavin Marx, Marco Matos, Louise Nott, Nick Pavlakis, Alex Powell, Timothy Price, David Ransom, Eva Segelov, Jenny Shannon, Nimit Singhal, Andrew Strickland, Euan Walpole: Belgium, Michel Craninx, Thierry Delaunoit, Amélie Deleporte, Karen Geboes, Michel Ferrante, Marc de Man, Els Monsaert, Veerle Moons, Marc Peeters, Marc Polus: France, Eveline Boucher, Jacques Balosso, Patrick Chevallier, Samy Louafi, Christine Rebischung, Denis Smith, Julien Taieb, Eric Terrebonne: Germany, Harald-Robert Bruch, Gerald Gehbauer, Volker Heinemann, Thomas Helmberger, Yon-Dschun Ko, Hendrik Kröning, Frank Lammert, Stefan Pluntke, Arnd Nusch, Karsten Ridwelski, Hanno Riess, Jorge Ramon Riera, Jens Ricke, Tilmann Sauerbruch, Klemens Scheidhauer, Oliver Stötzer, Klaus Tatsch, Ursula Vehling-Kaiser, Thomas Vogl: Italy, Bruna Angelelli: Israel, Alex Beny, Ravit Geva, Einat Shacham-Shmueli, Salomon Stemmer, Thomas Tichler, Ido Wolf: New Zealand, Michael P.N. Findlay, Richard Isaacs, Anne O’Donnell, David Perez, Bridget A. Robinson: Spain, Javier Rodríguez, Ruth Vera-Garcia: USA, Pradip Amin, Daniel Bloomgarden, James Bui, James Carlisle, Yi-Jen Chen, Andrew Coveler, Francis Facchini, Gary Frenette, Jacob Frick, Michael Garofalo, Benjamin George, Michael Gordon, Seza Gulec, James Hannigan, Matthew Holtzman, Andreas Kaubisch, Todd Kooy, Jeffrey Margolis, Robert Martin, Howard Ozer, Siddarth Padia, William Rilling, Michael Savin, Elyse Schneiderman, Grant Seeger, Navesh Sharma, Stephen Shibata, Randall Smith, Eric Wang, Samuel Whiting. 


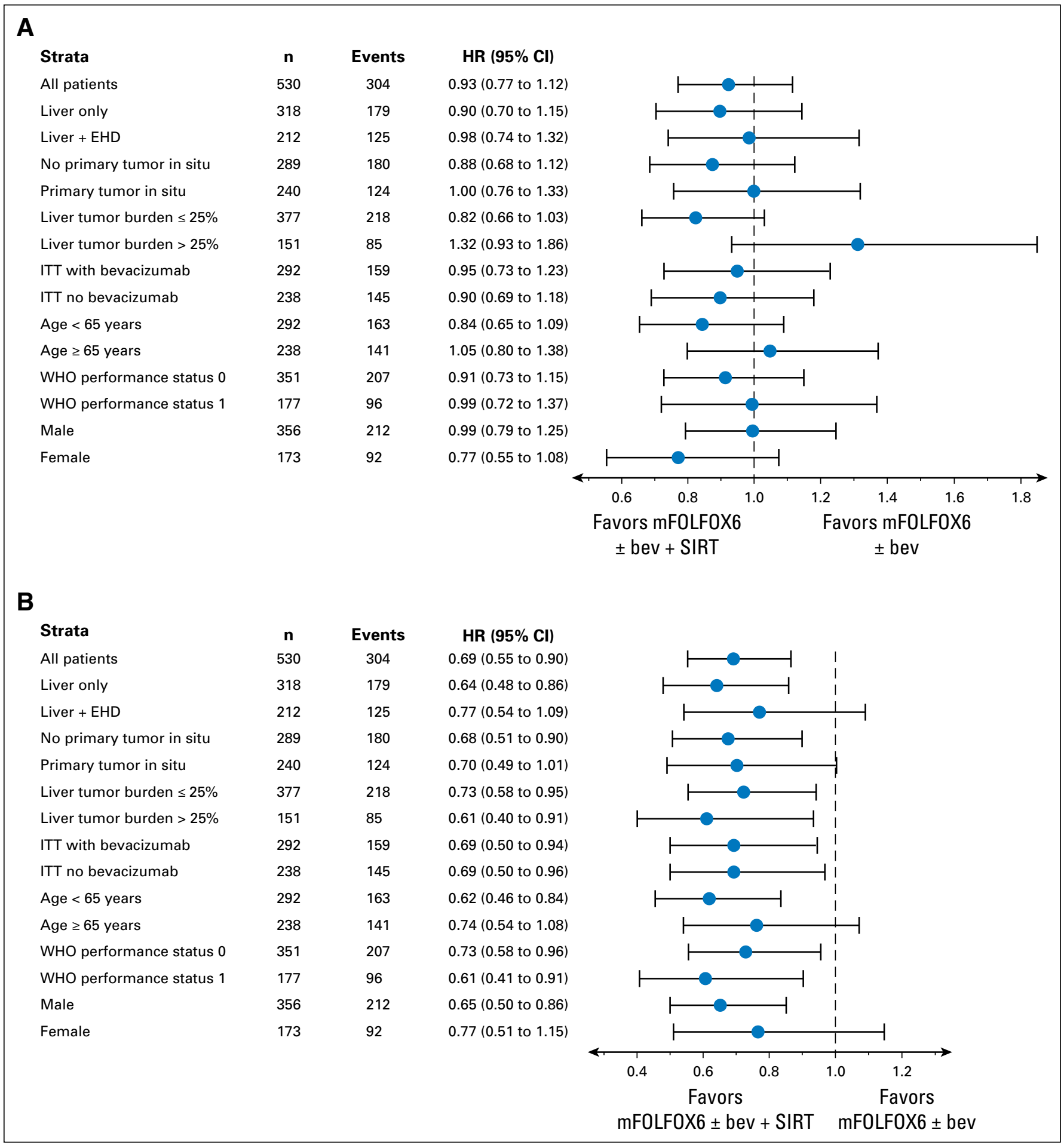

Fig A1. (A) Forest plot of planned subgroup analyses of progression-free survival at any site (ITT population). (B) Forest plot of planned subgroup analyses of progression

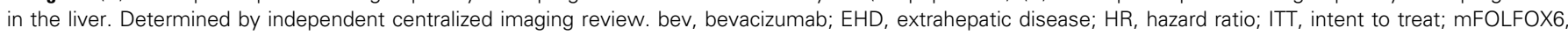
modified fluorouracil, leucovorin, and oxaliplatin; SIRT, selective internal radiation therapy. 
Table A1. Patient Eligibility Criteria for SIRFLOX Study

Inclusion Criteria

Written informed consent provided

$\geq 18$ years old with histologically confirmed adenocarcinoma of the colon or rectum (with or without the primary tumor in situ)

Proven liver metastases

WHO performance status of 0 to 1

Life expectancy of $\geq 3$ months

Patients with additional limited extrahepatic metastases in the lung (fewer than five nodules of $\leq 1 \mathrm{~cm}$ diameter or a single nodule of $\leq 1.7 \mathrm{~cm}$

diameter) and/or lymph node involvement in a single anatomic area of $<2 \mathrm{~cm}$ diameter) with the aim of these patients being $<40 \%$ of the total number of patients recruited (but not being excluded even if they account for more than this proportion).

Chemotherapy naïve for $\mathrm{mCRC}$, but previous adjuvant systemic chemotherapy for primary $\mathrm{CRC}$ or neoadjuvant chemoradiotherapy to the pelvis $>6$ months before recruitment are permitted

Deemed suitable for either treatment regimen by the investigator

Adequate hematologic, renal, and hepatic function

Using an acceptable method of contraception

\section{Exclusion Criteria}

Evidence of ascites, cirrhosis, portal hypertension, main portal venous tumor involvement, or main portal venous thrombosis

Previous radiation therapy to the upper abdomen

Nonmalignant disease that renders patients unsuitable for the study treatment

Grade $>1$ peripheral neuropathy ( $\mathrm{NCl}-\mathrm{CTC} 3$ )

Previous dose-limiting toxicity associated with adjuvant FU or oxaliplatin chemotherapy

Pregnancy or breast-feeding

Current or history of cancer other than adequately treated nonmelanoma skin cancer or carcinoma in situ of the cervix

Allergy to nonionic contrast agents.

Abbreviations: CRC, colorectal cancer; FU, fluorouracil; mCRC, metastatic colorectal cancer; NCl-CTCv3, National Cancer Institute Common Toxicity Criteria version 3 .

\begin{tabular}{|c|c|c|c|}
\hline $\begin{array}{c}\text { Number of First Progressions and Pattern of } \\
\text { First Disease Progression }\end{array}$ & $\begin{array}{c}\text { Control Arm } \\
\text { (mFOLFOX6 }[ \pm \text { bev]) }\end{array}$ & $\begin{array}{c}\text { Treatment Arm } \\
(\mathrm{SIRT}+\mathrm{mFOLFOX6}[ \pm \text { bev }])\end{array}$ & $P$ \\
\hline Number of first progressions & 178 & 166 & \\
\hline \multicolumn{4}{|l|}{ Pattern of first disease progression } \\
\hline Any site & & & $<.001$ \\
\hline Existing lesions & $118(66.3)$ & $68(41.0)$ & \\
\hline New lesions & $22(12.4)$ & 57 (34.3) & \\
\hline Existing + new lesions & $38(21.3)$ & $41(24.7)$ & \\
\hline Liver ( \pm other sites) & & & $<.001$ \\
\hline Existing lesions & $129(72.5)$ & $80(48.2)$ & \\
\hline New lesions & $10(5.6)$ & $24(14.5)$ & \\
\hline Existing + new lesions & $25(14.0)$ & $16(9.6)$ & \\
\hline Lung ( \pm other sites) & & & .273 \\
\hline Existing lesions & $5(2.8)$ & $8(4.8)$ & \\
\hline New lesions & $28(15.7)$ & $54(32.5)$ & \\
\hline Existing + new lesions & $1(0.6)$ & $9(5.4)$ & \\
\hline Lymph nodes ( \pm other sites) & & & .596 \\
\hline Existing lesions & $3(1.7)$ & $3(1.8)$ & \\
\hline New lesions & $4(2.2)$ & $7(4.2)$ & \\
\hline Existing + new lesions & $0(0.0)$ & $0(0.0)$ & \\
\hline \multicolumn{4}{|l|}{ Abdominal wall ( \pm other sites) } \\
\hline Existing lesions & $0(0.0)$ & $0(0.0)$ & \\
\hline New lesions & $0(0.0)$ & $1(0.6)$ & \\
\hline Existing + new lesions & $0(0.0)$ & $0(0.0)$ & \\
\hline
\end{tabular}

NOTE. Data are presented as No. (\%).

Abbreviations: bev, bevacizumab; mFOLFOX6, modified fluorouracil, leucovorin, and oxaliplatin; SIRT, selective internal radiation therapy. 
Table A3. Objective Response According to RECIST v1.0 at Any Site and in the Liver (ITT Population) as Assessed by Independent Readers

\begin{tabular}{|c|c|c|c|}
\hline Responses & $\begin{array}{c}\text { Control Arm } \\
\text { (mFOLFOX6 [ } \pm \text { bev }])\end{array}$ & $\begin{array}{c}\text { Treatment Arm } \\
(\mathrm{SIRT}+\mathrm{mFOLFOX6}[ \pm \text { bev }])\end{array}$ & $P$ \\
\hline ITT Population & 263 & 267 & \\
\hline \multicolumn{4}{|l|}{ Response at any site } \\
\hline Objective response (CR + PR) & $179(68.1)$ & $204(76.4)$ & .113 \\
\hline Complete response (CR) & $4(1.5)$ & $12(4.5)$ & .054 \\
\hline Partial response (PR) & $175(66.5)$ & 192 (71.9) & \\
\hline Stable disease & 48 (18.3) & $28(10.5)$ & \\
\hline Progressive disease & $17(6.5)$ & $25(9.4)$ & \\
\hline Not evaluable & $19(7.2)$ & $10(3.7)$ & \\
\hline \multicolumn{4}{|l|}{ Response in the liver } \\
\hline Objective response (CR $+\mathrm{PR})$ & $181(68.8)$ & $210(78.7)$ & .042 \\
\hline Complete response (CR) & $5(1.9)$ & $16(6.0)$ & .020 \\
\hline Partial response (PR) & $176(66.9)$ & $194(72.7)$ & \\
\hline Stable disease & 47 (17.9) & 29 (10.9) & \\
\hline Progressive disease & $16(6.1)$ & $18(6.7)$ & \\
\hline Not evaluable & $19(7.2)$ & $10(3.7)$ & \\
\hline
\end{tabular}

